\title{
Solutions of Zhiber-Shabat and Related Equations Using a Modified tanh-coth Function Method
}

\author{
Luwai Wazzan \\ Department of Mathematics, King Abdulaziz University, Jeddah, Saudi Arabia \\ Email: Iwazzan@hotmail.com
}

Received 20 February 2016; accepted 13 June 2016; published 16 June 2016

Copyright (C) 2016 by author and Scientific Research Publishing Inc.

This work is licensed under the Creative Commons Attribution International License (CC BY). http://creativecommons.org/licenses/by/4.0/

c) (i) Open Access

\section{Abstract}

The modified tanh-coth function method is used to obtain new exact travelling wave solutions for Zhiber-Shabat equation and the related equations: Liouville equation, sinh-Gordon equation, Dodd-Bullough-Mikhailov equation, and Tzitzeica-Dodd-Bullough equation. Exact travelling wave solutions for each equation are derived and expressed in terms of hyperbolic functions, trigonometric functions and rational functions. The modified tanh-coth function method is easy to execute, brief, efficient, and can be used to solve many other nonlinear evolution equations.

\section{Keywords}

A Modified tanh-coth Function Method, Zhiber-Shabat Equation, Liouville Equation, sinh-Gordon Equation, Dodd-Bullough-Mikhailov Equation, Tzitzeica-Dodd-Bullough Equation, Travelling Wave Solutions, Solitary Wave Solutions

\section{Introduction}

In this study we will investigate the solution of the nonlinear Zhiber-Shabat equation [1]

$$
u_{x t}+p \mathrm{e}^{u}+q \mathrm{e}^{-u}+r \mathrm{e}^{-2 u}=0
$$

where $p, q$ and $r$ are arbitrary constants. If $q=r=0$, Equation (1) becomes the Liouville equation. If $r=0$, Equation (1) becomes the sinh-Gordon equation. And for $q=0$, Equation (1) reduces to the well-known DoddBullough-Mikhailov equation. However, for $p=0, q=-1, r=1$, we get the Tzitzeica-Dodd-Bullough equation. These equations play an important role in many areas such as solid state physics, nonlinear optics, plasma physics, fluid dynamics, mathematical biology, nonlinear optics, dislocation in crystals, kink dynamics, and quantum 
filed theory [1]. The Zhiber-Shabat equation and other related equations were studied by some authors. Wazwaz in [2] and [3] applied the tanh method and the extended tanh method for handling the Zhiber-Shabat equation and other related equations: Liouville equation, sinh-Gordon, Dodd-Bullough-Mikhailov equation, and TzitzeicaDodd-Bullough equation. Fan and Hon in [4] have used the extended tanh method for handling Dodd-BulloughMikhailov equation. Wu and He in [5] solved the Dodd-Bullough-Mikhailov equation using the Exp-function method. Wazzan in [6] solved the Zhiber-Shabat equation and other related equations using the $\left(\frac{G^{\prime}}{G}\right)$ expansion-method. Our intention in this work is to find new solitary wave solutions for the nonlinear Zhiber-Shabat equation. Since there is no unified method that can be used to handle all types of nonlinear problems, we will use a modified tanh-coth function method [7]-[10]. Moreover, we will carry out comparisons between solutions obtained by the modified tanh-coth function method and other aforementioned methods.

\section{The Modified tanh-coth Function Method}

\subsection{Description of the Method}

To illustrate the basic concepts of the modified tanh-coth function method, we consider a given PDE in two variables given by

$$
P\left(u, u_{t}, u_{x}, u_{t t}, u_{x t}, u_{x x}, \cdots\right)=0 .
$$

We first consider its travelling solutions $u=u(x, t)=u(\xi)$, where $\xi=x-\lambda t$ or $\xi=x+\lambda t$, then Equation (2) becomes an ordinary differential equation

$$
Q\left(u, u^{\prime}, u^{\prime \prime}, u^{\prime \prime \prime}, u^{\prime \prime \prime \prime}, \cdots\right)=0 .
$$

The next crucial step is that the solution we are looking for is expressed in the form:

$$
u(\xi)=a_{0}+\sum_{i=1}^{m}\left(a_{i} w^{i}+b_{i} w^{-i}\right)
$$

and

$$
w^{\prime}=R+w^{2},
$$

where $R$ is a parameter to be determined later, $w=w(\xi)$ and $w^{\prime}=\frac{d w}{d \xi}$. The parameter $m$ can be found by balancing the highest order linear term with the nonlinear terms. Inserting (4) and (5) into the ordinary differential Equation (3) will yield a system of algebraic equations with respect to $a_{0}, a_{i}, b_{i}$ and $R$ (where $i=1, \cdots, m$ ). Because all the coefficients of $w^{i}$ have to vanish, and using any symbolic computation program such as Maple or Mathematica, one can determine $a_{0}, a_{i}, b_{i}$ and $R$. The Riccati Equation (5) has the following general solutions:

1) If $R<0$,

$$
\begin{gathered}
w=-\sqrt{-R} \tanh [\sqrt{-R} \xi], \\
w=-\sqrt{-R} \operatorname{coth}[\sqrt{-R} \xi] .
\end{gathered}
$$

2) If $R=0$,

$$
w=\frac{1}{\xi} .
$$

3) If $R>0$,

$$
\begin{gathered}
w=\sqrt{R} \tan [\sqrt{R} \xi], \\
w=-\sqrt{R} \cot [\sqrt{R} \xi] .
\end{gathered}
$$

In the next section, five examples in mathematical physics are chosen to illustrate the modified tanh-coth 
function method.

\subsection{Application}

\subsubsection{The Zhiber-Shabat Equation}

As before, we use $u(x, t)=u(\xi)$ where $\xi=x-\lambda t$, this will carry out the Zhiber-Shabat Equation (1) into

$$
-c u_{\xi \xi}+p \mathrm{e}^{u}+q \mathrm{e}^{-u}+r \mathrm{e}^{-2 u}=0 .
$$

We use the Painleve property:

$$
v=\mathrm{e}^{u},
$$

or equivalently

$$
u=\ln v,
$$

from which we find

$$
\begin{gathered}
u^{\prime}=\frac{1}{v} v^{\prime}, \\
u^{\prime \prime}=\frac{1}{v} v^{\prime \prime}-\frac{1}{v^{2}}\left(v^{\prime}\right)^{2} .
\end{gathered}
$$

The transformations (7) and (8) carry out (6) into the ODE

$$
-c\left(v v^{\prime \prime}-\left(v^{\prime}\right)^{2}\right)+p v^{3}+q v+r=0 .
$$

Using the modified tanh-coth function method, balancing the term $v v^{\prime \prime}$ with $v^{3}$, gives $m=2$, hence we set the modified tanh-coth function method assumption as follows:

$$
v(x, t)=v(\xi)=a_{0}+a_{1} w+a_{2} w^{2}+\frac{b_{1}}{w}+\frac{b_{2}}{w^{2}}
$$

where

$$
w^{\prime}=R+w^{2},
$$

and

$$
w^{\prime \prime}=2 R w+2 w^{3} .
$$

Without loss of generality, we set $p=q=r=1$.

Substituting (10) into (9), and making use of Equation (11) collecting the coefficients of each power of $w$, and using Maple to solve the nonlinear system in $a_{0}, a_{1}, a_{2}, b_{1}, b_{2}$ and $R$, we obtain:

1) First set

$$
a_{0}=\frac{\gamma}{12 \alpha}, b_{2}=0, a_{2}=2 \lambda, R=\frac{-1}{2 \lambda}\left(\frac{\gamma^{2}-15 \alpha \gamma-36 \alpha^{2}}{36 \alpha^{2}}\right) .
$$

2) Second set

$$
\begin{gathered}
a_{0}=\frac{\gamma}{12 \alpha}, a_{2}=0, b_{2}=\frac{1}{2 \lambda}\left(\frac{3 \gamma^{2}-16 \alpha \gamma-144 \alpha^{2}}{36 \alpha^{2}}\right), \\
R=\frac{2 b_{2}}{31}\left(\frac{11 \gamma^{2}-78 \alpha \gamma-504 \alpha^{2}}{36 \alpha^{2}}\right) .
\end{gathered}
$$

3) Third set

$$
a_{0}=\frac{-11}{3596}\left(\frac{\bar{\gamma}^{2}+810 \overline{\alpha \gamma}+25668 \bar{\alpha}^{2}}{36 \bar{\alpha}^{2}}\right), a_{2}=2 \lambda, b_{2}=\frac{1}{128 \lambda}\left(\frac{\bar{\gamma}}{6 \bar{\alpha}}\right),
$$




$$
R=\frac{1}{496 \lambda}\left(\frac{\bar{\gamma}^{2}-6 \overline{\alpha \gamma}-1116 \bar{\alpha}^{2}}{36 \bar{\alpha}^{2}}\right)
$$

4) Fourth set

$$
\begin{gathered}
a_{0}=\frac{-11}{14384}\left(\frac{\bar{\beta}^{2}-1620 \bar{\beta} \bar{\alpha}-115632 \bar{\alpha}^{2}}{36 \bar{\alpha}^{2}}\right), a_{2}=2 \lambda, b_{2}=\frac{-\bar{\beta}}{1536 \lambda \bar{\alpha}}, \\
R=\frac{1}{1984 \lambda}\left(\frac{\bar{\beta}^{2}+12 \bar{\beta} \bar{\alpha}-4464 \bar{\alpha}^{2}}{36 \bar{\alpha}^{2}}\right) .
\end{gathered}
$$

5) Fifth set

$$
a_{0}=\frac{-\beta}{24 \alpha}, a_{2}=2 \lambda, b_{2}=0, R=\frac{-1}{8 \lambda}\left(\frac{\beta^{2}+30 \beta \alpha-144 \alpha^{2}}{36 \alpha^{2}}\right) .
$$

6) Sixth set

$$
\begin{gathered}
a_{0}=\frac{-\beta}{24 \alpha}, a_{2}=0, b_{2}=\frac{1}{32 \lambda}\left(\frac{9 \beta^{2}+96 \beta \alpha-1720 \alpha^{2}}{36 \alpha^{2}}\right), \\
R=\frac{b_{2}}{62}\left(\frac{11 \beta^{2}+156 \beta \alpha-2016 \alpha^{2}}{36 \alpha^{2}}\right),
\end{gathered}
$$

where,

$$
\begin{gathered}
\alpha=(188+12 \sqrt{93})^{\frac{1}{3}}, \\
\gamma=\alpha^{2}+2 \alpha+28, \\
\bar{\alpha}=(106028+10788 \sqrt{93})^{\frac{1}{3}}, \\
\bar{\gamma}=\bar{\alpha}^{2}+2 \bar{\alpha}+748, \\
\beta=(\gamma-6 \alpha) \pm i \sqrt{3}\left(\alpha^{2}-28\right), \\
\bar{\beta}=(\bar{\gamma}-6 \bar{\alpha}) \pm i \sqrt{3}\left(\bar{\alpha}^{2}-748\right) .
\end{gathered}
$$

Note that, using the numerical value of $\alpha$, we deduce $\gamma^{2}-15 \alpha \gamma-36 \alpha^{2}=\alpha^{4}-11 \alpha^{3}-6 \alpha^{2}-308 \alpha+784<0$.

Recall that $u(x, t)=\ln (v(x, t))$, hence we obtain:

According to the first set, for $R<0$, solutions for Equation (6) read

$$
u_{1}(x, t)=\ln \left\{\frac{\gamma}{12 \alpha}+\frac{\gamma^{2}-15 \alpha \gamma-36 \alpha^{2}}{36 \alpha^{2}} \tanh ^{2}\left[\frac{1}{12 \alpha} \sqrt{\frac{2\left(\gamma^{2}-15 \alpha \gamma-36 \alpha^{2}\right)}{\lambda}}(x-\lambda t)\right]\right\}
$$

and

$$
u_{2}(x, t)=\ln \left\{\frac{\gamma}{12 \alpha}+\frac{\gamma^{2}-15 \alpha \gamma-36 \alpha^{2}}{36 \alpha^{2}} \operatorname{coth}^{2}\left[\frac{1}{12 \alpha} \sqrt{\frac{2\left(\gamma^{2}-15 \alpha \gamma-36 \alpha^{2}\right)}{\lambda}}(x-\lambda t)\right]\right\} .
$$

However, for $R>0$, the solutions are

$$
u_{3}(x, t)=\ln \left\{\frac{\gamma}{12 \alpha}-\frac{\gamma^{2}-15 \alpha \gamma-36 \alpha^{2}}{36 \alpha^{2}} \tan ^{2}\left[\frac{1}{12 \alpha} \sqrt{-\frac{2\left(\alpha^{4}-11 \alpha^{3}-6 \alpha^{2}-308 \alpha+784\right)}{\lambda}}(x-\lambda t)\right]\right\} \text {, }
$$


and

$$
u_{4}(x, t)=\ln \left\{\frac{\gamma}{12 \alpha}+\frac{\gamma^{2}-15 \alpha \gamma-36 \alpha^{2}}{36 \alpha^{2}} \cot ^{2}\left[\frac{1}{12 \alpha} \sqrt{-\frac{2\left(\alpha^{4}-11 \alpha^{3}-6 \alpha^{2}-308 \alpha+784\right)}{\lambda}}(x-\lambda t)\right]\right\} \text {. }
$$

According to second set, notice that $11 \gamma^{2}-78 \alpha \gamma-504 \alpha^{2}=11 \alpha^{4}-34 \alpha^{3}-952 \alpha+8624>0$ and $3 \gamma^{2}-16 \alpha \gamma-144 \alpha^{2}>0$, using the numerical value of $\alpha$, and for $R<0$, we obtain the solutions:

$$
\begin{aligned}
& u_{5}(x, t)=\ln \left\{\frac{\gamma}{12 \alpha}-\frac{558 \alpha^{2}}{11 \gamma^{2}-78 \alpha \gamma-504 \alpha^{2}} \tanh ^{2}\left[\frac{1}{6 \alpha} \sqrt{-\frac{2\left(11 \gamma^{2}-78 \alpha \gamma-504 \alpha^{2}\right) b_{2}}{31}}(x-\lambda t)\right]\right\}, \\
& u_{6}(x, t)=\ln \left\{\frac{\gamma}{12 \alpha}-\frac{558 \alpha^{2}}{11 \gamma^{2}-78 \alpha \gamma-504 \alpha^{2}} \operatorname{coth}^{2}\left[\frac{1}{6 \alpha} \sqrt{-\frac{2\left(11 \gamma^{2}-78 \alpha \gamma-504 \alpha^{2}\right) b_{2}}{31}}(x-\lambda t)\right]\right\} .
\end{aligned}
$$

However, for $R>0$, we obtain the travelling wave solutions:

$$
u_{7}(x, t)=\ln \left\{\frac{\gamma}{12 \alpha}+\frac{558 \alpha^{2}}{11 \gamma^{2}-78 \alpha \gamma-504 \alpha^{2}} \tan ^{2}\left[\frac{1}{6 \alpha} \sqrt{\frac{2\left(11 \gamma^{2}-78 \alpha \gamma-504 \alpha^{2}\right) b_{2}}{31}}(x-\lambda t)\right]\right\}
$$

and

$$
u_{8}(x, t)=\ln \left\{\frac{\gamma}{12 \alpha}+\frac{558 \alpha^{2}}{11 \gamma^{2}-78 \alpha \gamma-504 \alpha^{2}} \cot ^{2}\left[\frac{1}{6 \alpha} \sqrt{\frac{2\left(11 \gamma^{2}-78 \alpha \gamma-504 \alpha^{2}\right) b_{2}}{31}}(x-\lambda t)\right]\right\},
$$

where $b_{2}$ is given in 2).

According to The third set, notice that $\bar{\gamma}^{2}-6 \overline{\alpha \gamma}-1116 \bar{\alpha}^{2}>0$, using the numerical value of $\alpha$, we obtain the soliton solutions, for $R<0$,

$$
\begin{aligned}
u_{9}(x, t)= & \ln \left\{\frac{-11}{3596}\left(\frac{\bar{\gamma}^{2}+810 \overline{\alpha \gamma}+25668 \bar{\alpha}^{2}}{36 \bar{\alpha}^{2}}\right)-\frac{1}{248}\left(\frac{\bar{\gamma}^{2}-6 \overline{\alpha \gamma}-1116 \bar{\alpha}^{2}}{36 \bar{\alpha}^{2}}\right)\right. \\
& \left.\times \tanh ^{2}[\sqrt{-R}(x-\lambda t)]-\frac{93 \overline{\alpha \gamma}}{4\left(\bar{\gamma}^{2}-6 \overline{\alpha \gamma}-1116 \bar{\alpha}^{2}\right)} \operatorname{coth}^{2}[\sqrt{-R}(x-\lambda t)]\right\} .
\end{aligned}
$$

However, for $R>0$, we obtain the travelling wave solutions:

$$
\begin{aligned}
u_{10}(x, t)= & \ln \left\{\frac{-11}{3596}\left(\frac{\bar{\gamma}^{2}+810 \overline{\alpha \gamma}+25668 \bar{\alpha}^{2}}{36 \bar{\alpha}^{2}}\right)+\frac{1}{248}\left(\frac{\bar{\gamma}^{2}-6 \overline{\alpha \gamma}-1116 \bar{\alpha}^{2}}{36 \bar{\alpha}^{2}}\right)\right. \\
& \left.\times \tan ^{2}[\sqrt{R}(x-\lambda t)]+\frac{93 \overline{\alpha \gamma}}{4\left(\bar{\gamma}^{2}-6 \overline{\alpha \gamma}-1116 \bar{\alpha}^{2}\right)} \cot ^{2}[\sqrt{R}(x-\lambda t)]\right\},
\end{aligned}
$$

where, $R$ is given in 3).

Note that, $u_{1}, u_{2}, u_{3}$ and $u_{4}$ are also obtained by Wazwaz using the tanh-function method in [2]. Other solutions are not reported in [2].

Sets of solutions in 4)-6) will give complex solutions.

\subsubsection{The Liouville Equation}

As stated before, if $q=r=0, p=1$ in the Zhiber-Shabat Equation (1), we obtain the Liouville equation:

$$
u_{x t}+\mathrm{e}^{u}=0 .
$$


Using the wave variable $\xi=x-\lambda t$, we get

$$
-c u_{\xi \xi}+\mathrm{e}^{u}=0 .
$$

We again use the Painleve property:

$$
u=\ln v,
$$

to transform (12) into the ODE

$$
-c\left(v v^{\prime \prime}-\left(v^{\prime}\right)^{2}\right)+v^{3}=0 .
$$

Considering the homogeneous balance between $v v^{\prime \prime}$ and $v^{3}$ in Equation (13), gives $m=2$, and using the modified tanh-coth function method, we suppose that the solution of Equation (13) is in the form:

$$
v(x, t)=v(\xi)=a_{0}+a_{1} w+\frac{b_{1}}{w}+a_{2} w^{2}+\frac{b_{2}}{w^{2}} .
$$

Proceeding as before we found:

1) First set

$$
a_{0}=2 R \lambda, a_{1}=b_{1}=b_{2}=0, a_{2}=2 \lambda, R=R,
$$

2) Second set

$$
a_{0}=a_{1}=b_{1}=b_{2}=0, a_{2}=2 \lambda, R=0,
$$

3) Third set

$$
a_{0}=2 R \lambda, a_{1}=b_{1}=a_{2}=0, b_{2}=2 \lambda R^{2}, R=R,
$$

4) Fourth set

$$
a_{0}=4 R \lambda, a_{1}=b_{1}=0, a_{2}=2 \lambda, b_{2}=2 \lambda R^{2}, R=R,
$$

where $R$ is free parameter. Recall that $u(x, t)=\ln v(x, t)$.

According to the first set we obtain the solutions:

$$
\begin{gathered}
u_{1}(x, t)=\ln \left\{2 \lambda R\left(1-\tanh ^{2}[\sqrt{-R}(x-\lambda t)]\right)\right\}, R<0, \\
u_{2}(x, t)=\ln \left\{2 \lambda R\left(1-\operatorname{coth}^{2}[\sqrt{-R}(x-\lambda t)]\right)\right\}, R<0, \\
u_{3}(x, t)=\ln \left\{2 \lambda R\left(1+\tan ^{2}[\sqrt{R}(x-\lambda t)]\right)\right\}, R>0, \\
u_{4}(x, t)=\ln \left\{2 \lambda R\left(1+\cot ^{2}[\sqrt{R}(x-\lambda t)]\right)\right\}, R>0, \\
u_{5}(x, t)=\ln \left\{\frac{2 \lambda}{(x-\lambda t)^{2}}\right\}, R=0 .
\end{gathered}
$$

According to the third set we obtain the similar to the solutions of the first set.

According to the fourth set we obtain the solutions, $R<0$,

$$
u_{6}(x, t)=\ln \left\{2 \lambda R\left(1-\tanh ^{2}[\sqrt{-R}(x-\lambda t)]-\operatorname{coth}^{2}[\sqrt{-R}(x-\lambda t)]\right)\right\},
$$

and if $R>0$, then

$$
u_{7}(x, t)=\ln \left\{2 \lambda R\left(1+\tan ^{2}[\sqrt{R}(x-\lambda t)]+\cot ^{2}[\sqrt{R}(x-\lambda t)]\right)\right\} .
$$

Note that, $u_{1}$ and $u_{2}$ are also obtained by Wazwaz using the tanh-function method in [2]. Other solutions are not reported in [2]. 


\subsubsection{The sinh-Gordon Equation}

As stated before, if $r=0, q=1, p=1$ in the Zhiber-Shabat Equation (1), we obtain the sinh-Gorden equation:

$$
u_{x t}+\mathrm{e}^{u}+\mathrm{e}^{-u}=0 .
$$

Using the wave variable $\xi=x-\lambda t$, we get

$$
-c u_{\xi \xi}+\mathrm{e}^{u}-\mathrm{e}^{-u}=0,
$$

Using the Painleve property, Equation (14) is transformed into the ODE

$$
-c\left(v v^{\prime \prime}-\left(v^{\prime}\right)^{2}\right)+v^{3}-v=0 .
$$

The balancing process gives $m=2$. We can suppose that the solution of Equation (15) is the form:

$$
v(x, t)=v(\xi)=a_{0}+a_{2} w^{2}+\frac{b_{2}}{w^{2}} .
$$

Following the same analysis presented above, we obtain:

1) First set

$$
a_{0}=0, a_{2}=2 \lambda=\frac{ \pm 1}{R}, b_{2}=0, R= \pm \frac{1}{2 \lambda} .
$$

2) Second set

$$
a_{0}=0, a_{2}=0, b_{2}=\frac{1}{2 \lambda}= \pm R, R= \pm \frac{1}{2 \lambda} .
$$

3) Third set

$$
a_{0}=\frac{ \pm 1}{2}, a_{2}=2 \lambda=\frac{ \pm 1}{4 R}, b_{2}=\frac{1}{32 \lambda}, R= \pm \frac{1}{8 \lambda} .
$$

According to the first set, and for $R<0$, we obtain

$$
\begin{aligned}
& u_{1}(x, t)=\ln \left\{\tanh ^{2}\left[\sqrt{\frac{1}{2 \lambda}}(x-\lambda t)\right]\right\}, \lambda>0, \\
& u_{2}(x, t)=\ln \left\{-\tanh ^{2}\left[\sqrt{\frac{-1}{2 \lambda}}(x-\lambda t)\right]\right\}, \lambda<0, \\
& u_{3}(x, t)=\ln \left\{\operatorname{coth}^{2}\left[\sqrt{\frac{1}{2 \lambda}}(x-\lambda t)\right]\right\}, \lambda>0, \\
& u_{4}(x, t)=\ln \left\{-\operatorname{coth}^{2}\left[\sqrt{\frac{-1}{2 \lambda}}(x-\lambda t)\right]\right\}, \lambda<0,
\end{aligned}
$$

for $R>0$, we obtain

$$
\begin{aligned}
& u_{5}(x, t)=\ln \left\{\tan ^{2}\left[\sqrt{\frac{1}{2 \lambda}}(x-\lambda t)\right]\right\}, \lambda>0, \\
& u_{6}(x, t)=\ln \left\{-\tan ^{2}\left[\sqrt{\frac{-1}{2 \lambda}}(x-\lambda t)\right]\right\}, \lambda<0, \\
& u_{7}(x, t)=\ln \left\{\cot ^{2}\left[\sqrt{\frac{1}{2 \lambda}}(x-\lambda t)\right]\right\}, \lambda>0,
\end{aligned}
$$




$$
u_{8}(x, t)=\ln \left\{-\cot ^{2}\left[\sqrt{\frac{-1}{2 \lambda}}(x-\lambda t)\right]\right\}, \lambda<0 .
$$

According to the second set, we obtain similar solutions to the solutions of the first set.

According to the third set, we obtain, for $R<0, \lambda>0$,

$$
u_{9,10}(x, t)=\ln \left\{ \pm \frac{1}{2}\left(1+\frac{1}{2} \tanh ^{2}\left[\frac{1}{2} \sqrt{\frac{1}{2 \lambda}}(x-\lambda t)\right]+\frac{1}{2} \operatorname{coth}^{2}\left[\frac{1}{2} \sqrt{\frac{1}{2 \lambda}}(x-\lambda t)\right]\right)\right\} \text {, }
$$

for $R<0$,

$$
u_{11}(x, t)=\ln \left\{-\frac{1}{2}\left(1+\frac{1}{2} \tanh ^{2}\left[\frac{1}{2} \sqrt{\frac{-1}{2 \lambda}}(x-\lambda t)\right]+\frac{1}{2} \operatorname{coth}^{2}\left[\frac{1}{2} \sqrt{\frac{-1}{2 \lambda}}(x-\lambda t)\right]\right)\right\}
$$

for $R>0$,

$$
u_{12}(x, t)=\ln \left(-\frac{1}{2}\left(1-\frac{1}{2} \tan ^{2}\left[\frac{1}{2} \sqrt{\frac{1}{2 \lambda}}(x-\lambda t)\right]-\frac{1}{2} \cot ^{2}\left[\frac{1}{2} \sqrt{\frac{1}{2 \lambda}}(x-\lambda t)\right]\right),\right.
$$

and for $R>0$,

$$
u_{13}(x, t)=\ln \left(\frac{1}{2}\left(1-\frac{1}{2} \tan ^{2}\left[\frac{1}{2} \sqrt{\frac{-1}{2 \lambda}}(x-\lambda t)\right]-\frac{1}{2} \cot ^{2}\left[\frac{1}{2} \sqrt{\frac{-1}{2 \lambda}}(x-\lambda t)\right]\right)\right) .
$$

Note that, $u_{1}$ and $u_{3}$ are also obtained by Wazwaz using the tanh-function method in [2]. Other solutions are not reported in [2].

\subsubsection{The Dodd-Bullough-Mikhailov Equation}

If $p=1, q=0, r=1$ in the Zhiber-Shabat Equation (1), we obtain the Dodd-Bullough-Mikhailov equation:

$$
u_{x t}+\mathrm{e}^{u}+\mathrm{e}^{-2 u}=0,
$$

and by using the wave variable $\xi=x-\lambda t$, we get

$$
-c u_{\xi \xi}+\mathrm{e}^{u}+\mathrm{e}^{-2 u}=0 .
$$

We use the Painleve property:

$$
u=\ln v,
$$

to transform (17) into the ODE

$$
-c\left(v v^{\prime \prime}-\left(v^{\prime}\right)^{2}\right)+v^{3}+1=0
$$

Considering the homogeneous balance between $v v^{\prime \prime}$ and $v^{3}$ in Equation (18), gives $m=2$, we can suppose that the solution of Equation (15) is the form

$$
v(x, t)=v(\xi)=a_{0}+a_{1} w+\frac{b_{1}}{w}+a_{2} w^{2}+\frac{b_{2}}{w^{2}} .
$$

Proceeding as before, we get

1) First set

2) Second set

$$
a_{0}=\frac{1}{2}, a_{1}=b_{1}=b_{2}=0, a_{2}=2 \lambda, R=\frac{3}{4 \lambda} .
$$

$$
a_{0}=\frac{1}{2}\left(-\frac{1}{2} \mp \frac{i}{2} \sqrt{3}\right), a_{1}=b_{1}=b_{2}=0, a_{2}=2 \lambda, R=\frac{3}{4} \frac{-\frac{1}{2} \mp \frac{i}{2} \sqrt{3}}{\lambda} .
$$


3) Third set

$$
a_{0}=\frac{1}{2}, a_{1}=b_{1}=a_{2}=0, b_{2}=\frac{9}{8 \lambda}, R=\frac{3}{4 \lambda} .
$$

4) Fourth set

$$
a_{0}=\frac{1}{2}\left(-\frac{1}{2} \mp \frac{i}{2} \sqrt{3}\right), a_{1}=b_{1}=a_{2}=0, b_{2}=-\frac{9}{8} \frac{\frac{1}{2} \mp \frac{i}{2} \sqrt{3}}{\lambda}, R=\frac{3}{4} \frac{-\frac{1}{2} \mp \frac{i}{2} \sqrt{3}}{\lambda} .
$$

5) Fifth set

$$
a_{0}=-\frac{1}{4}, a_{1}=b_{1}=0, a_{2}=2 \lambda, b_{2}=\frac{9}{128 \lambda}, R=\frac{3}{16 \lambda} .
$$

6) Sixth set

$$
a_{0}=\frac{1}{4}\left(\frac{1}{2} \pm \frac{i}{2} \sqrt{3}\right), a_{1}=b_{1}=0, a_{2}=2 \lambda, b_{2}=\frac{-9}{128} \frac{\frac{1}{2} \mp \frac{i}{2} \sqrt{3}}{\lambda}, R=\frac{3}{16} \frac{-\frac{1}{2} \mp \frac{i}{2} \sqrt{3}}{\lambda} .
$$

According to the first set,we obtain the soliton solutions:

$$
u_{1}(x, t)=\ln \left\{\frac{1}{2}\left(1-3 \tanh ^{2}\left[\frac{1}{2} \sqrt{\frac{-3}{\lambda}}(x-\lambda t)\right]\right)\right\}, \lambda<0,
$$

and

$$
u_{2}(x, t)=\ln \left\{\frac{1}{2}\left(1-3 \operatorname{coth}^{2}\left[\frac{1}{2} \sqrt{\frac{-3}{\lambda}}(x-\lambda t)\right]\right)\right\}, \lambda<0,
$$

for $\lambda>0$, we obtain the travelling wave solutions:

$$
u_{3}(x, t)=\ln \left\{\frac{1}{2}\left(1+3 \tan ^{2}\left[\frac{1}{2} \sqrt{\frac{3}{\lambda}}(x-\lambda t)\right]\right)\right\},
$$

and

$$
u_{4}(x, t)=\ln \left\{\frac{1}{2}\left(1+3 \cot ^{2}\left[\frac{1}{2} \sqrt{\frac{3}{\lambda}}(x-\lambda t)\right]\right)\right\} .
$$

According to the second set, we obtain the solutions

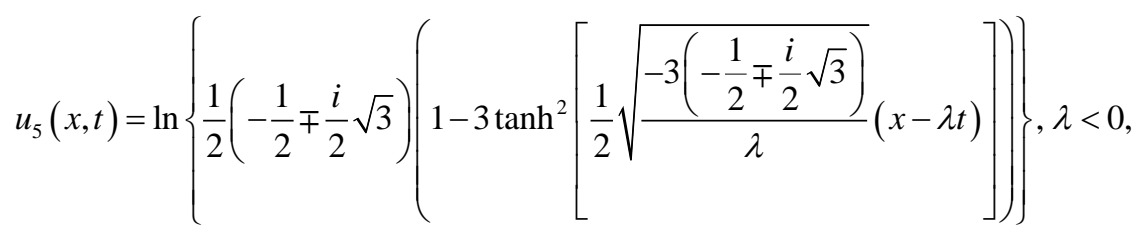

and

$$
u_{6}(x, t)=\ln \left\{\frac{1}{2}\left(-\frac{1}{2} \mp \frac{i}{2} \sqrt{3}\right)\left(1-3 \operatorname{coth}^{2}\left[\frac{1}{2} \sqrt{\frac{-3\left(-\frac{1}{2} \mp \frac{i}{2} \sqrt{3}\right)}{\lambda}}(x-\lambda t)\right]\right), \lambda<0,\right.
$$

for $\lambda>0$, we obtain the travelling wave solutions: 


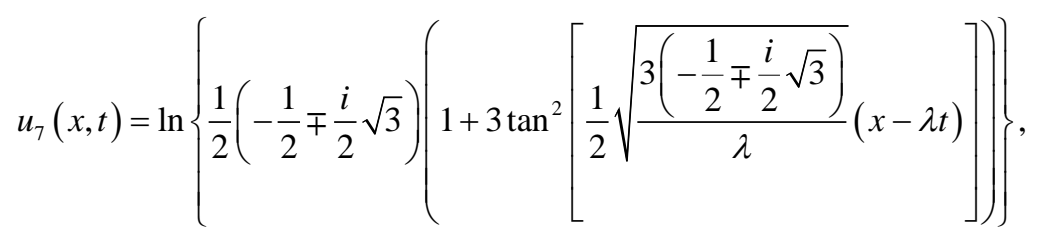

and

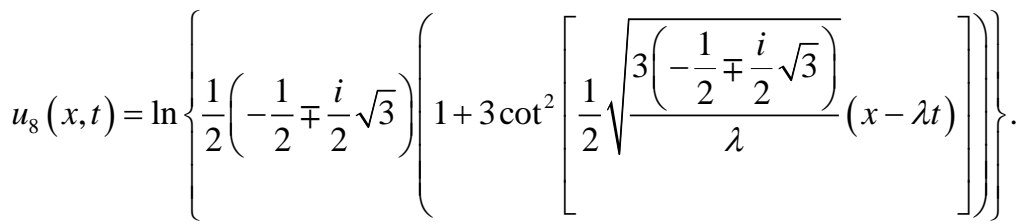

According to the third set and fourth set, we obtain similar solutions to the solutions of the first set and second set, respectively.

According to fifth set, we obtain, for $\lambda<0$, the following solutions

$$
u_{9}=\ln \left\{\frac{-1}{4}\left(1+\frac{3}{2} \tanh ^{2}\left[\frac{1}{4} \sqrt{\frac{-3}{\lambda}}(x-\lambda t)\right]+\frac{3}{2} \operatorname{coth}^{2}\left[\frac{1}{4} \sqrt{\frac{-3}{\lambda}}(x-\lambda t)\right]\right)\right\},
$$

for $\lambda>0$, we obtain the travelling wave solutions

$$
u_{10}=\ln \left\{\frac{-1}{4}\left(1-\frac{3}{2} \tan ^{2}\left[\frac{1}{4} \sqrt{\frac{3}{\lambda}}(x-\lambda t)\right]-\frac{3}{2} \cot ^{2}\left[\frac{1}{4} \sqrt{\frac{3}{\lambda}}(x-\lambda t)\right]\right)\right\} .
$$

According to sixth set, for $\lambda>0$, this in turn gives the solitons solutions:

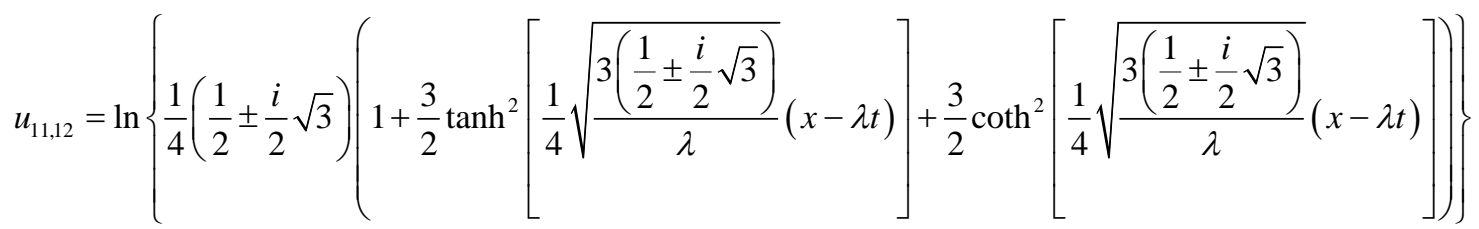

for $\lambda<0$, we obtain the travelling wave solutions:

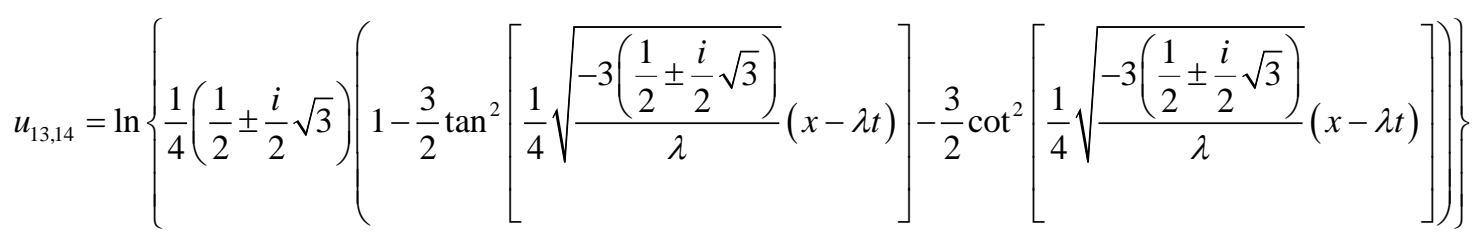

The solutions $u_{1}, u_{3}, u_{3}, u_{4}, u_{9}$, and $u_{10}$ are also obtained by Wazwaz using the tanh-function method in [3]. Other solutions are not reported in [3].

\subsubsection{The Tzitzeica-Dodd-Bullough Equation}

This equation can be obtained if we set $p=0, q=-1, r=1$ in the Zhiber-Shabat Equation (1), and by using the wave variable $\xi=x-\lambda t$, we find the Tzitzeica-Dodd-Bullough equation:

$$
-c u_{\xi \xi}-\mathrm{e}^{-u}+\mathrm{e}^{-2 u}=0,
$$

suppose that

$$
v=\mathrm{e}^{-u}
$$

or equivalently

$$
u=\ln \left(\frac{1}{v}\right)
$$


By using (23) we can transform Equation (21) to

$$
c\left(v v^{\prime \prime}-\left(v^{\prime}\right)^{2}\right)-v^{3}+v^{4}=0 .
$$

Considering the homogeneous balance between $v v^{\prime \prime}$ and $v^{4}$ in Equation (24), gives $m=1$, and by using the modified tanh-coth function method we can suppose that the solution of Equation (24) is the form:

$$
v(x, t)=v(\xi)=a_{0}+a_{1} w+\frac{b_{1}}{w} .
$$

Proceeding as before, we get the following set of solutions.

1) First set

2) Second set

$$
a_{0}=\frac{1}{2}, a_{1}= \pm \sqrt{\frac{-1}{4 R}}, b_{1}=0, \lambda=\frac{1}{4 R}
$$

3) Third set

$$
a_{0}=\frac{1}{2}, a_{1}=0, b_{1}= \pm \sqrt{\frac{-1}{4 R}}, \lambda=\frac{1}{4 R}
$$

$$
a_{0}=\frac{1}{2}, a_{1}=\sqrt{\frac{-1}{16 R}}, b_{1}= \pm \sqrt{\frac{-R}{16}}, \lambda=\frac{1}{16 R}
$$

According to the first set, we obtain the solitons solutions

$$
u_{1,2}(x, t)=\ln \left(\frac{2}{1 \pm \tanh \left[\sqrt{-R}\left(x-\frac{1}{4 R} t\right)\right]}\right), R<0,
$$

and

$$
u_{3,4}(x, t)=\ln \left(\frac{2}{1 \pm \operatorname{coth}\left[\sqrt{-R}\left(x-\frac{1}{4 R} t\right)\right]}\right), R<0,
$$

for $R>0$, we obtain the solutions:

$$
u_{5,6}(x, t)=\ln \left(\frac{2}{1 \pm i \tan \left[\sqrt{R}\left(x-\frac{1}{4 R} t\right)\right]}\right)
$$

and

$$
u_{7,8}(x, t)=\ln \left(\frac{2}{1 \pm i \cot \left[\sqrt{R}\left(x-\frac{1}{4 R} t\right)\right]}\right)
$$

According to the second set, we obtain the solutions

$$
u_{9,10}(x, t)=\ln \left(\frac{2}{1 \pm \frac{1}{2}\left(\tanh \left[\sqrt{-R}\left(x-\frac{1}{16 R} t\right)\right]+\operatorname{coth}\left[\sqrt{-R}\left(x-\frac{1}{16 R} t\right)\right]\right)}\right), R<0 .
$$


For $R>0$, we obtain the solutions:

$$
u_{11,12}(x, t)=\ln \left(\frac{2}{1 \pm \frac{1}{2}\left(i \tan \left[\sqrt{-R}\left(x-\frac{1}{16 R} t\right)\right]-i \cot \left[\sqrt{-R}\left(x-\frac{1}{16 R} t\right)\right]\right)}\right) .
$$

According to the third set, we obtain the solutions

$$
u_{13,14}=u_{9,10}, u_{15,16}=u_{11,12} \text {. }
$$

The solutions $u_{1,2}, u_{3,4}, u_{5,6}$ and $u_{7,8}$ are also obtained by Wazwaz using the tanh-function method in [3]. Other solutions are not reported in [3].

\section{Conclusion}

The Zhiber-Shabat equation, and the related equations: Liouville equation, sinh-Gordon equation Dodd-BulloughMikhailov equation, and the Tzitzeica-Dodd-Bullough equation were investigated using a modified tanh-coth method. New travelling wave solutions were established. The modified tanh-coth function method is a robust computational tool for obtaining exact solutions for the nonlinear Zhiber-Shabat equation, and the related equations. It is also an encouraging method to solve other nonlinear evolution equations.

\section{References}

[1] Sirendaoreji, J.S. (2002) A Direct Method for Solving sinh-Gordon Type Equation. Physics Letters A, 298, $133-139$. http://dx.doi.org/10.1016/S0375-9601(02)00513-3

[2] Wazwaz, A.M. (2008) The tanh Method for Travelling Wave Solutions to the Zhiber-Shabat Equation and Other Related Equations. Communications in Nonlinear Science and Numerical Simulation, 13, 584-592. http://dx.doi.org/10.1016/j.cnsns.2006.06.014

[3] Wazwaz, A.M. (2005) The tanh Method: Solitons and Periodic Solutions for the Dodd-Bullough-Mikhailov and the Tzitzeica-Dodd-Bullough Equations. Chaos, Solitons and Fractals, 25, 55-63. http://dx.doi.org/10.1016/j.chaos.2004.09.122

[4] Fan, F. and Hon, Y.C. (2003) Applications of Extended tanh Method to "Special” Types of Nonlinear Equations. Applied Mathematics and Computation, 141, 351-358. http://dx.doi.org/10.1016/S0096-3003(02)00260-6

[5] He, J.-H. and Wu, X.-H. (2006) Exp-Function Method for Nonlinear Wave Equations. Chaos, Solitons and Fractals, 30, 700-708. http://dx.doi.org/10.1016/j.chaos.2006.03.020

[6] Wazzan, L. (2015) New Exact Travelling Wave Solutions of the Nonlinear Zhiber-Shabat Equation. Far East Journal of Applied Mathematics, 90, 213-244. http://dx.doi.org/10.17654/FJAMMar2015_213_244

[7] El-Wakil, S.A., El-labany, S.K., Zahran, M.A. and Sabry, R. (2005) Modified Extended tanh-Function Method and Its Applications to Nonlinear Equations. Applied Mathematics and Computation, 161, 403. http://dx.doi.org/10.1016/j.amc.2003.12.035

[8] El-Wakil, S.A., El-labany, S.K., Zahran, M.A. and Sabry, R. (2002) Modified Extended tanh-Function Method for Solving Nonlinear Partial Differential Equations. Physics Letters A, 299, 179. http://dx.doi.org/10.1016/S0375-9601(02)00669-2

[9] Wazwaz, A.M. (2006) The tanh and the sine-cosine Methods for a Reliable Treatment of the Modified Equal Width Equation and Its Variants. Communications in Nonlinear Science and Numerical Simulation, 11, 148-160. http://dx.doi.org/10.1016/j.cnsns.2004.07.001

[10] Wazzan, L. (2009) A Modified tanh-coth Method for Solving the General Burgers-Fisher and the Kuramoto-Sivashinsky Equations. Communications in Nonlinear Science and Numerical Simulation, 14, 2642-2652. http://dx.doi.org/10.1016/j.cnsns.2008.08.004 